\title{
Brittle-to-ductile transition in a fiber bundle with strong heterogeneity
}

\author{
Kornél Kovács, ${ }^{1}$ Raul Cruz Hidalgo, ${ }^{2}$ Ignacio Pagonabarraga,${ }^{3}$ and Ferenc Kun ${ }^{1, *}$ \\ ${ }^{1}$ Department of Theoretical Physics, University of Debrecen, P. O. Box: 5, H-4010 Debrecen, Hungary \\ ${ }^{2}$ Departamento de Fisica, Facultad de Ciencias, Universidad de Navarra, 31080 Pamplona, Spain \\ ${ }^{3}$ Departament de Física Fonamental, Universitat de Barcelona, Carrer Martí i Franqués 1, E-08028 Barcelona, Spain
}

(Received 20 December 2012; published 29 April 2013)

\begin{abstract}
We analyze the failure process of a two-component system with widely different fracture strength in the framework of a fiber bundle model with localized load sharing. A fraction $0 \leqslant \alpha \leqslant 1$ of the bundle is strong and it is represented by unbreakable fibers, while fibers of the weak component have randomly distributed failure strength. Computer simulations revealed that there exists a critical composition $\alpha_{c}$ which separates two qualitatively different behaviors: Below the critical point, the failure of the bundle is brittle, characterized by an abrupt damage growth within the breakable part of the system. Above $\alpha_{c}$, however, the macroscopic response becomes ductile, providing stability during the entire breaking process. The transition occurs at an astonishingly low fraction of strong fibers which can have importance for applications. We show that in the ductile phase, the size distribution of breaking bursts has a power law functional form with an exponent $\mu=2$ followed by an exponential cutoff. In the brittle phase, the power law also prevails but with a higher exponent $\mu=\frac{9}{2}$. The transition between the two phases shows analogies to continuous phase transitions. Analyzing the microstructure of the damage, it was found that at the beginning of the fracture process cracks nucleate randomly, while later on growth and coalescence of cracks dominate, which give rise to power law distributed crack sizes.
\end{abstract}

DOI: 10.1103/PhysRevE.87.042816

PACS number(s): 46.50.+a, 05.90.+m, 81.40.Np, 64.60.A-

\section{INTRODUCTION}

Beyond its engineering relevance, the complexity of the fracture of brittle and ductile materials is of great interest for physics and materials science, as well. Recently, the application of statistical physics has revealed interesting novel aspects of the damage and fracture of heterogeneous materials increasing our understanding both on the microscales and macroscales of fracture processes [1]. Most of these theoretical investigations rely on mesoscopic discrete models such as fiber bundles [2-6] and lattices of fuses [7,8], springs, or beams [9-11], where disorder is typically captured by the random strength of elements. Analytic calculations and computer simulations have revealed that for a broad class of disorder distributions, the fracture of heterogeneous materials exhibits universal aspects both on the microlevel and macrolevel: the size of bursts has a power law distribution with universal exponents [4-6,12,13], furthermore, macroscopic failure occurs in the form of localization after a precursory sequence of microcracking [14-16]. Interesting analogies have been established between the failure of materials and phase transitions and critical phenomena [2,16-19].

However, real materials are often of composite nature, i.e., they have two or more components with widely different properties which provide an improved fracture toughness. For instance, in fiber reinforced composites where fibers are embedded in a carrier matrix, the matrix material typically has much lower fracture strength than the fibers. This widely different strength of the components combined with appropriate coupling results in an improved damage tolerance which has a high relevance for applications [20,21]. It has been shown that in heterogeneous materials, varying the local mechanical response and of the amount of disorder of the components one

*ferenc.kun@science.unideb.hu can achieve a transition from brittle to quasibrittle or even to ductile failure [22-25].

In this paper, we study the effect of strong heterogeneity on the fracture process of disordered materials based on a fiber bundle model (FBM) with localized interaction. Our model is composed of two subsets of fibers with widely different fracture behavior: fibers of one of the subsets are strong in the sense that they can support any load and never break, while fibers of the other type are weak, characterized by a probability distribution of failure strength. The two components form a homogeneous mixture on a square lattice with periodic boundary conditions in both directions. It is a crucial element of our approach that after failure events the load of broken fibers is equally redistributed solely over their intact nearest neighbors, leading to high stress concentration around failed regions. Such localized load sharing systems are known to be very brittle, however, our investigations demonstrate that the presence of strong fibers moderates the effect of stress inhomogeneities and leads to a ductile macroscopic response when exceeding a critical fraction. Ductility is qualified in our system by the stable growth of cracks at the microscopic level, which leads to the emergence of a relatively long plateau regime in the macroscopic response of the system. We explore by computer simulations how the brittle-to-ductile transition occurs on the microscale by investigating the size distribution of bursts and the microstructure of damage.

\section{MIXTURE OF WEAK AND STRONG FIBERS}

We consider a parallel bundle of fibers organized on a square lattice of size $L$. Under an increasing external load, the fibers present a linearly elastic behavior characterized by the same Young modulus $E$. To model the two-component mixture, a fraction $0 \leqslant \alpha \leqslant 1$ of the $N=L^{2}$ fibers of the lattice is considered to be unbreakable, i.e., they can support any load without failure. These strong fibers of number $N_{s}=\alpha N$ are 
distributed randomly all over the lattice without any spatial correlations. The remaining $1-\alpha$ fraction of fibers of number $N_{w}=(1-\alpha) N$ are considered to be weak, i.e., they have only a finite load bearing capacity and break when the local load on them exceeds their failure threshold. The breaking thresholds of weak fibers $\sigma_{t h}^{i}, i=1, \ldots, N_{w}$, are independent, identically distributed random variables with a probability density $p\left(\sigma_{t h}\right)$ and distribution function $P\left(\sigma_{t h}\right)$. For simplicity, we consider a uniform distribution between 0 and 1 so that $p\left(\sigma_{t h}\right)$ and $P\left(\sigma_{t h}\right)$ take the forms $p\left(\sigma_{t h}\right)=1$ and $P\left(\sigma_{t h}\right)=\sigma_{t h}$, respectively. The bundle is subject to a quasistatically increasing external load $\sigma$ parallel to the direction of fibers. When a fiber breaks in the bundle, its load has to be shared by the remaining intact fibers. As a crucial element of the model, we assume that the load sharing is completely localized in the system so that the excess load after failure events is equally redistributed over the intact nearest neighbors of failed fibers. This localized load sharing (LLS) introduces spatial correlations in the system, which makes it impossible to carry out analytical calculations.

\section{A. Equal load sharing (ELS)}

In a previous work [26], we have shown that in the limit case of equal load sharing (ELS), the most important characteristics of the fracture process of the two-component system can be obtained in closed analytical form. Under ELS conditions, the constitutive relation $\sigma^{\mathrm{ELS}}(\varepsilon)$ of the model can be written as

$$
\sigma^{\mathrm{ELS}}(\varepsilon)=(1-\alpha)[1-P(E \varepsilon)] E \varepsilon+\alpha E \varepsilon,
$$

where $\varepsilon$ denotes the strain of the system. The first term of Eq. (1) accounts for the load bearing capacity of the surviving fraction of weak elements, and the second one represents the stress carried by the unbreakable subset of the system. This constitutive equation $\sigma^{\mathrm{ELS}}(\varepsilon)$ is illustrated in Fig. 1 as continuous curves for uniformly distributed failure thresholds at several values of $\alpha$. Note that one recovers the usual FBM constitutive behavior [13] in the limiting case of $\alpha=0$, when the bundle is only composed of weak fibers. Those solutions usually present a parabolic maximum, which defines the critical deformation $\varepsilon_{c}^{\text {ELS }}$ and strength $\sigma_{c}^{\text {ELS }}$ under stress-controlled conditions [26]. For finite values of $\alpha$, all

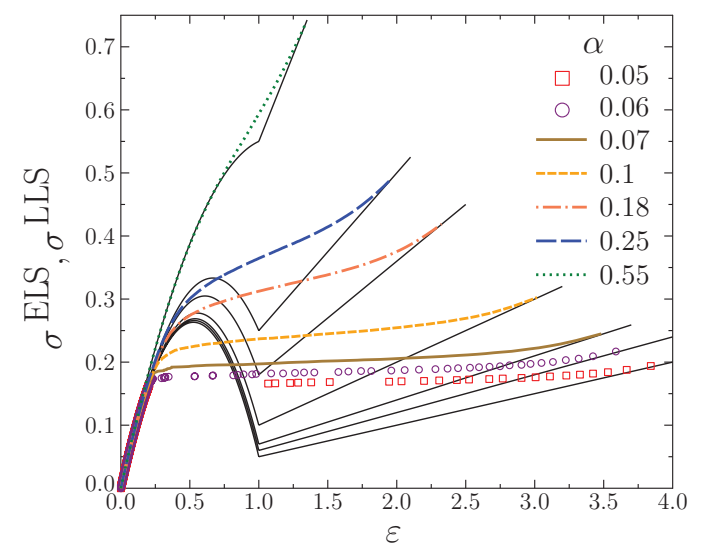

FIG. 1. (Color online) The constitutive behavior of the twocomponent bundle for ELS (black continuous lines) and LLS (dasheddotted lines with different colors). The transition from brittle to ductile behavior can clearly be observed as $\alpha$ increases from bottom to top. the weak fibers break for large enough $\varepsilon$ so that the first term of Eq. (1) goes to zero while the unbreakable fibers overtake the entire external load. Consequently, the constitutive curves in Fig. 1 tend asymptotically to straight lines with slope $\alpha E$. It can be seen in Fig. 1 that for low values of $\alpha$, the local maximum of $\sigma^{\mathrm{ELS}}(\varepsilon)$ prevails but its position $\varepsilon_{c}^{\mathrm{ELS}}$ and value $\sigma_{c}^{\text {ELS }}$ are monotonically increasing with $\alpha$ [26].

For the case of a uniform distribution, we have analytically found that the position of the maximum depends on $\alpha$ following $\sigma_{c}^{\mathrm{ELS}}(\alpha)=1 / 2(1-\alpha)$ [26]. This holds for $\alpha \leqslant$ $\alpha_{c}^{\text {ELS }}$ with the critical value of the control parameter $\alpha_{c}^{\text {ELS }}=\frac{1}{2}$. At $\alpha_{c}^{\text {ELS }}$, the value of $\varepsilon_{c}^{\text {ELS }}$ coincides with the upper bound of strength values $\sigma_{t h}^{\max } / E=1$. The parabolic shape of the constitutive curve prevails even for $\alpha>\alpha_{c}^{\text {ELS }}$, but $\sigma^{\text {ELS }}(\varepsilon)$ becomes linear at $\varepsilon=\sigma_{t h}^{\max } / E$ before reaching the maximum, so that the rest of the parabola can not be realized. Additionally, it was also shown analytically that the existence of this critical point and the qualitatively different shape of the constitutive curve below and above $\alpha_{c}^{\text {ELS }}$ have a substantial effect on the microscopic breaking of the system [26].

\section{B. Local load sharing (LLS)}

In this work, we examine the case of localized load redistribution. In the following, we show that the presence of unbreakable fibers results in a broad spectrum of behaviors when the stress field is inhomogeneous. In the two-component mixture, the unbreakable fibers act as a load reservoir, i.e., the load they carry, especially the load increments they receive from their broken weak neighbors, does not contribute to the breaking process; from the viewpoint of breakable fibers, this load is dissipated. It has the consequence that increasing the fraction of strong fibers reduces the stress concentration around failed regions. The value of $\alpha$ controls the relevance of the stress concentration and the disorder during the failure process. Thus, it has interesting consequences on both the microscale and macroscale responses of the system.

In order to obtain a detailed understanding of the effect of stress localization on the fracture process in two-component mixtures, we carried out computer simulations on a square lattice of size $L=401$ with periodic boundary conditions in both directions, varying the value of $\alpha$ between 0 and 1 . Averages were calculated over 100 samples with different realizations of disorder.

Figure 1 illustrates the constitutive curve of the LLS bundle $\sigma^{\text {LLS }}(\varepsilon)$ for several values of $\alpha$ in comparison with the corresponding ELS results $\sigma^{\mathrm{ELS}}(\varepsilon)$. At $\alpha=0$ when the system contains only breakable fibers, we recover the former LLS results, namely, the LLS constitutive curve follows the ELS analytic solution [3,13,27-31]. However, the critical stress $\sigma_{c}^{\text {LLS }}$ and strain $\varepsilon_{c}^{\text {LLS }}$, where macroscopic failure occurs, are significantly lower than the ELS values $\sigma_{c}^{\text {ELS }}$ and $\varepsilon_{c}^{\text {ELS }}$ falling in the linear regime of the constitutive curve. This result implies that under LLS conditions, FBMs exhibit a more brittle behavior than for long range load redistribution. Under stress-controlled loading, macroscopic failure occurs in the form of a catastrophic avalanche at $\sigma_{c}^{\text {LLS }}$ during which all remaining fibers break. For finite $\alpha$ values, it can be observed in Fig. 1 that the structure of $\sigma^{\mathrm{LLS}}(\varepsilon)$ has two substantially different regimes: increasing the external load $\sigma$ for $\alpha$ values 
close to zero the constitutive curve has a finite horizontal jump, then it continues again along the ELS analytic solution. The discontinuity of $\sigma^{\mathrm{LLS}}(\varepsilon)$ is the consequence of a major avalanche in which a macroscopic fraction of weak fibers breaks. However, not all the weak fibers do break at this large event. The smooth convergence of $\sigma^{\mathrm{LLS}}(\varepsilon)$ to the asymptotic linear curve indicates that a small fraction of fibers survives and gradually breaks as the external load $\sigma$ is further increased. It is important to emphasize that there exists a well defined value $\alpha_{c}$ of the control parameter at which the jump and the dominating avalanche disappear and $\sigma^{\text {LLS }}(\varepsilon)$ becomes a continuous monotonically increasing function. Increasing $\alpha$ above $\alpha_{c}$, the qualitative form of the constitutive curve does not change anymore. It is a very important feature of the system that in the regime $\alpha>\alpha_{c}$, the macroscopic response exhibits ductile behavior, i.e., instead of the abrupt damage growth observed below the critical point, the fracture process retains stability up to very large strain values. This brittle-to-ductile transition occurs at an astonishingly low fraction of strong fibers $\alpha_{c}^{\text {LLS }} \approx 0.059(5)$ compared to the corresponding meanfield counterpart $\alpha_{c}^{\mathrm{LLS}} \ll \alpha_{c}^{\mathrm{ELS}}$.

In local load sharing approximation, the critical strength $\sigma_{c}$ of a homogeneous system of breakable fibers $(\alpha=0)$ depends logarithmically on the system size. Consequently, $\sigma_{c}$ vanishes in the thermodynamic limits. However, for $\alpha>$ 0 , when increasing the system size, the weight of both components (breakable and unbreakable) increases with the same proportion. Furthermore, in the thermodynamic limits, one could expect a similar logarithmic convergence but to a finite value $\sigma_{c}(\alpha)$. Therefore, we expect that the system size dependence of $\sigma_{c}(\alpha)$ will not significantly modify the shape of the curves shown in Fig. 1, as well as the value of $\alpha_{c}^{\mathrm{LLS}}$.

The unique macroscopic response of the system is the fingerprint of the special features of the microscopic fracture process. Due to the localized interaction of fibers, both spatial and temporal correlations arise at the micro-level: breaking fibers trigger bursts which are spatially localized and result in correlated growth of cracks. In order to characterize the breaking process on the microscale, we analyze the statistics of bursts of simultaneously failing fibers and the spatial structure of broken clusters.

\section{BURST SIZE DISTRIBUTION}

The failure process of LLS fiber bundles has recently been explored in detail by computer simulations in the case of $\alpha=0$ where the bundle is only composed of weak fibers $[3,13,21$, 27-31]. It was found that under an increasing external load, first the weakest fibers break randomly and homogeneously over the entire system. As the external load increases, the load dropped by the broken fibers becomes sufficient to give rise to additional breakings. As a result, breaking fibers can trigger avalanches of breaking events which are spatially correlated giving rise to growing clusters of broken elements. Connected clusters of broken fibers on the lattice can be interpreted as cracks in FBMs [21,28]. The microscopic origin of the brittle behavior, observed in the limit of $\alpha=0$ in Fig. 1, is that along the perimeter of growing cracks a high load is concentrated, which easily initiates a catastrophic avalanche already at crack sizes much smaller than the system size. As a consequence, in

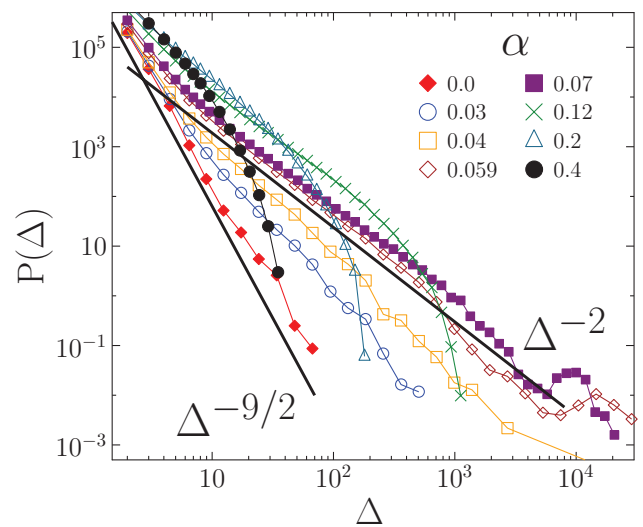

FIG. 2. (Color online) Avalanche size distributions $P(\Delta)$ at different values of $\alpha$ below and above $\alpha_{c}$. Straight lines with slope $\frac{9}{2}$ and 2.0 are drawn to guide the eye. The value of $\alpha$ increases from bottom to top in the range $\Delta<10$.

our simulations the completely weak system can not tolerate large bursts and the avalanche size distribution $P(\Delta)$ becomes a rapidly decreasing function. The data analysis show that the distribution function is a power law with a relatively large exponent (see Fig. 2)

$$
P(\Delta) \sim \Delta^{-\mu}, \quad \text { where } \quad \mu \approx 9 / 2,
$$

in agreement with former predictions [27,28,31]. For increasing $\alpha$, simulations revealed that the reduction of stress concentration by strong fibers allows the system for larger avalanches. It can be observed in Fig. 2 that the exponent $\mu \approx \frac{9}{2}$ still remains for small avalanche sizes, while for large avalanches a crossover occurs to a lower exponent $\mu \approx 2.0$. Approaching the critical value $\alpha_{c}$, the second power law regime spans over nearly three orders of magnitude in a system of size $L=401$. Note the bump of $P(\Delta)$ for the largest avalanches. Since the largest avalanche of the system is always followed by a few small ones, no catastrophic event can be identified. Consequently, all the avalanches were taken into account when performing the counting. Hence, the statistics of the largest avalanches forms a bump of Gaussian shape on the total distribution. Figure 2 shows that above $\alpha_{c}$ the bump in $P(\Delta)$, associated to large avalanches, disappears and an exponential cutoff develops instead. Increasing $\alpha$ further does not affect the power law distribution, although the cutoff avalanche size decreases.

Scaling analysis revealed that rescaling the two axes by appropriate powers of a characteristic avalanche size, the distributions $P(\Delta)$ above the critical point $\alpha_{c}$ can be collapsed onto a master curve. Using the average size of the largest avalanche $\bar{\Delta}_{\max }$ as scaling variable, an excellent quality data collapse is obtained in Fig. 3 which implies the scaling structure of the burst size distribution

$$
P(\Delta)=\bar{\Delta}_{\max }^{-\beta} f\left(\Delta / \bar{\Delta}_{\max }^{\xi}\right) \text { for } \alpha>\alpha_{c} .
$$

The best collapse is achieved for the exponents $\xi=1.24$ and $\beta=2.5$ as seen in Fig. 3. It is important to emphasize that the scaling function $f$ has the functional form

$$
f\left(\Delta / \bar{\Delta}_{\max }^{\xi}\right) \sim \Delta^{-\mu} \exp \left(-\Delta / \bar{\Delta}_{\max }^{\xi}\right),
$$




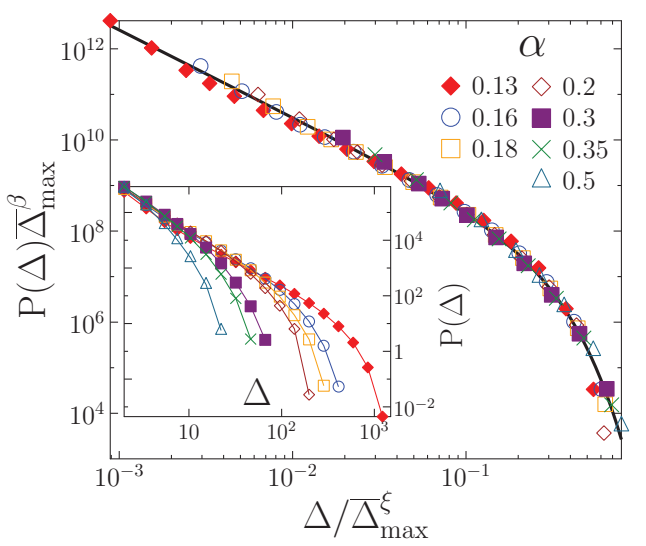

FIG. 3. (Color online) Scaling plot of avalanche size distributions above $\alpha_{c}$ using the average size of the largest avalanche $\bar{\Delta}_{\max }$ as scaling variable. An excellent collapse is achieved with the exponents $\xi=1.24$ and $\beta=2.5$. The bold line represents the fit with Eq. (4), where $\mu=1.97$ was obtained. The original distributions $P(\Delta)$ are presented in the inset where $\alpha$ increases from right to left.

i.e., it can be described as a power law followed by an exponential cutoff. The value of the exponent $\mu$ has been obtained by fitting as $\mu=2.0 \pm 0.05$. Note that due to the condition of normalization, the three exponents $\xi, \beta$, and $\mu$ must fulfill the scaling relation $\beta=\xi \mu$. By substituting the numerical values, good agreement can be found. The above scaling analysis also demonstrates that the value of the exponent $\mu$ of the burst size distribution is constant above the critical point so that any apparent change of $\mu$ in Fig. 3 can be attributed to the moving cutoff.

Computer simulations have shown that the characteristic avalanche size $\bar{\Delta}_{\max }$ as a function of $\alpha$ has a sharp peak (see Fig. 4) at the same $\alpha_{c}$ which has been defined based on the constitutive curve of the system in Fig. 1. Replotting $\bar{\Delta}_{\max }$ as a function of $\alpha-\alpha_{c}$ in the inset of Fig. 4, a straight line has been obtained on a double logarithmic plot which implies the power law form

$$
\bar{\Delta}_{\max } \sim\left(\alpha-\alpha_{c}\right)^{-\nu} \quad \text { for } \quad \alpha>\alpha_{c} .
$$

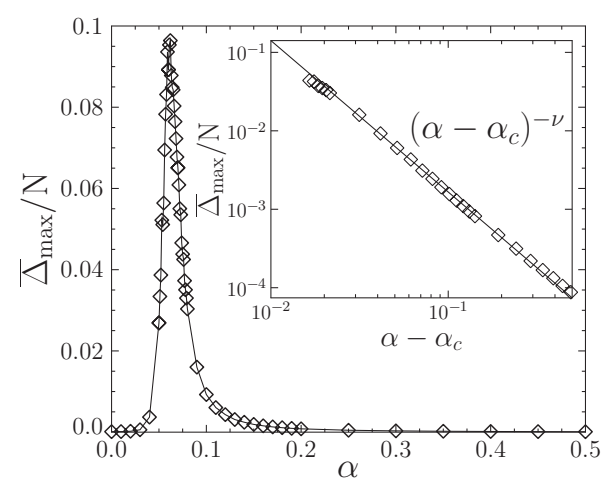

FIG. 4. Average size of the largest avalanche $\bar{\Delta}_{\max }$ as a function of $\alpha$. The position of the sharp peak $\alpha_{c}$ coincides with the critical value of $\alpha$ we identified based on the constitutive curves. Inset: the same quantity plotted as a function of the distance from the critical point $\alpha-\alpha_{c}$ for $\alpha>\alpha_{c}$. A straight line of slope 1.95 is drawn to guide the eye.
The value of the correlation length exponent $v$ was obtained numerically as $v=1.95 \pm 0.07$. The result shows that approaching the critical point $\alpha_{c}$ from above, the characteristic avalanche size has a power law divergence. When $\alpha$ is increased starting from zero, during the damaging of the bundle an increasing fraction of load is carried by the strong fibers. This induces a screening effect and part of the system becomes inaccessible for the damage growth. Consequently, the active load decreases and the bundle can survive larger avalanches, which reach the scale of the system size at $\alpha=\alpha_{c}$.

Comparing to the mean-field solution of the model [26], it is noticeable that the burst size exponent $\mu$ of the regime $\alpha \geqslant \alpha_{c}$ is smaller, while the correlation length exponent $v$ is larger when the stress redistribution is localized. In Ref. [12], we have shown by analytical means that the burst size exponent of FBMs takes the value $\mu=2$ when the constitutive curve has a long plateau preceded by an increasing regime with only a slight nonlinearity. Our numerical results obtained for two-component mixtures are in perfect agreement with the analytical predictions of Ref. [12].

\section{MICROSTRUCTURE OF DAMAGE}

Contrary to equal load sharing, under LLS conditions fibers breaking in a burst form a connected set which may be part of a more extended cluster of broken fibers generated by previous avalanches. Therefore, the cluster structure and the avalanches of breaking fibers become correlated. In the following, we explore how the microstructure of damage evolves under an increasing external load $\sigma$ for different compositions of the system and establish the relation of crack growth and breaking avalanches.

\section{A. Nucleation, growth, and merging of cracks}

It has been discussed that in the limit of $\alpha=0$, all clusters of broken fibers are small compared to the system size and they are randomly dispersed over the entire bundle. Macroscopic failure is typically driven by the breaking of a single fiber which is located along the boundary of a larger cluster where the stress concentration is high $[6,17,21,28,32,33]$. However, at finite values of $\alpha$, the presence of strong fibers substantially influences the cluster structure of broken fibers of the weak component: strong fibers decrease the load transferred to the weak ones after breaking events which reduce the stress concentration around failed regions. As a consequence, at low values of $\alpha$, strong fibers let the system grow larger avalanches, and hence, more extended clusters of broken fibers can occur. However, at high $\alpha$, strong fibers develop a counter effect, i.e., the load transferred to weak fibers after failure events will be so much reduced that it limits the propagating bursts and growing clusters. Since at a given value of $\alpha$ the bundle is a random mixture of weak and strong fibers, the underlying structural disorder of the mixture has also an additional effect. For the two-component system, a direct mapping can be established to percolation lattices where $p_{s}=\alpha$ and $p_{w}=1-\alpha$ are the occupation probabilities of strong and weak fibers, respectively, with $p_{s}+p_{w}=1$. In the regime $\alpha \leqslant p_{c}\left(p_{s} \leqslant p_{c}\right.$ and $\left.p_{w} \geqslant p_{c}\right)$ where $p_{c}$ corresponds to the critical occupation probability of site percolation on a square 

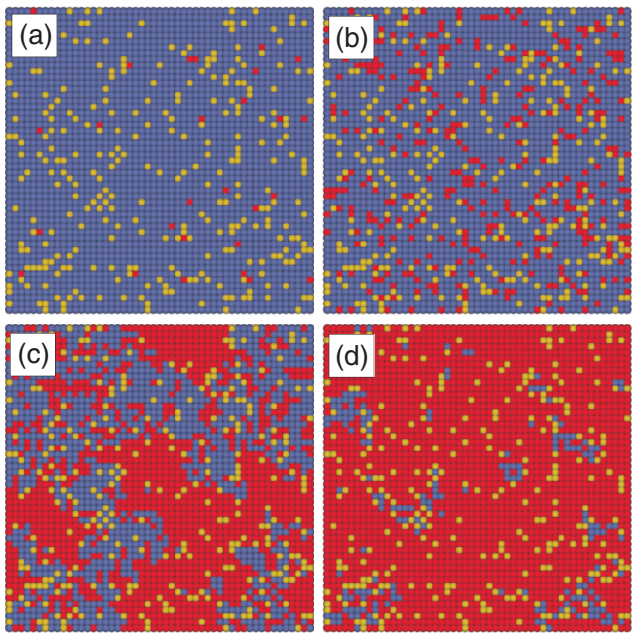

FIG. 5. (Color online) Snapshots of a damaging bundle of size $L=50$ at four values of $\varepsilon$ during the loading process with $\alpha=0.07$. Blue (gray) and yellow (light gray) colors represent intact weak and strong fibers, respectively, while red (dark gray) stands for the broken ones.

lattice [34], there exists a dominating cluster of weak fibers (spanning cluster). However, for $\alpha>p_{c}$, the strong fibers form a spanning cluster, while all weak clusters are small. The intermediate regime $\left(1-p_{c}\right)<\alpha<p_{c}$ is also interesting because here none of the components have a spanning cluster since $p_{w}<p_{c}$ and $p_{s}<p_{c}$ hold.

Figure 5 presents an example for the evolution of the cluster structure of the system for an increasing load $\sigma$ (for demonstration purposes, a relatively small lattice $L=50$ is considered). It can be seen in Fig. 5(a) that as the external load $\sigma$ is increased, randomly dispersed clusters of broken fibers grow. In the example, $\alpha=0.07$ is slightly above the critical point $\alpha_{c}$, hence, clusters become so large that they can also merge [see Figs. 5(b) and 5(c)]. Finally, in Fig. 5(d), nearly all weak fibers are broken so that cracks are identical with the clusters of the underlying weak component. It has been pointed out that the probability distribution of failure thresholds, especially the functional form of its tail, may also affect the cluster structure of broken fibers [21]. For simplicity, our analysis focuses solely on the case of uniformly distributed thresholds, however, the results on the micros-structure of damage may slightly change for other types of disorder.

In order to give a quantitative characterization of the evolution of the microstructure of damage we determined the average size $\left\langle S_{a v}\right\rangle$ and the average number $\left\langle n_{c}\right\rangle$ of clusters of broken fibers as a function of the deformation $\varepsilon$ during the loading process. The average cluster size $S_{a v}$ is defined as the ratio of the second and first moments of cluster sizes $S_{i}$,

$$
S_{a v}=\frac{\sum_{i} S_{i}^{2}}{\sum_{i} S_{i}},
$$

where the largest cluster is always omitted in the summation. Then, $\left\langle S_{a v}\right\rangle(\varepsilon)$ is obtained by averaging $S_{a v}$ over a large number of samples at the same deformation $\varepsilon$. It can be observed in Fig. 6(a) that in the brittle regime $\alpha<\alpha_{c}$ the average cluster size $\left\langle S_{a v}\right\rangle(\varepsilon)$ has only an increasing branch which suddenly stops at macroscopic failure. However, in the

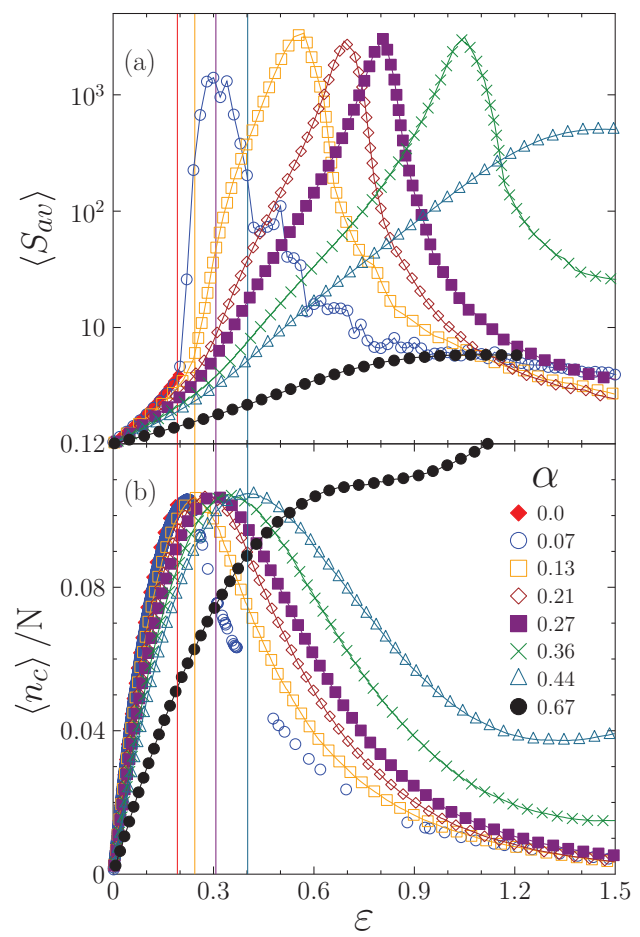

FIG. 6. (Color online) Average cluster size $\left\langle S_{a v}\right\rangle$ (a) and average number of clusters $\left\langle n_{c}\right\rangle$ (b) as function of $\varepsilon$ for several compositions $\alpha$ of the system. For clarity, the vertical straight lines indicate the position $\varepsilon_{m}^{n c}$ of the maximum of $\left\langle n_{c}\right\rangle$ for a few $\alpha$ values: $0.0,0.13,0.27,0.44$

ductile phase, the $\left\langle S_{a v}\right\rangle$ curves have a maximum followed by a rapidly decreasing part. Above the critical point $\alpha>\alpha_{c}$, the coalescence of cracks results in a macroscopic cluster which spans the entire system. The maximum of the $\left\langle S_{a v}\right\rangle$ curves occurring at the deformation $\varepsilon_{m}^{S}$ marks the configuration where the dominating cluster is formed. Since the largest cluster is always removed from the statistics, in the presence of a dominating cluster $\left\langle S_{a v}\right\rangle$ must decrease. The situation drastically changes when $\alpha$ surpasses the value of $\alpha^{*}=$ $1-p_{c} \approx 0.4077$ because in the regime $\alpha>\alpha^{*}$ the weak component does not develop a spanning cluster and hence no dominating crack can emerge. Consequently, in this regime, $\left\langle S_{a v}\right\rangle$ monotonically increases and saturates when nearly all weak fibers break.

It can be observed in Fig. 6(a) that the value of the maximum $\left\langle S_{a v}\right\rangle^{\text {max }}$ of the average cluster size shows also an interesting systematics as the composition $\alpha$ of the system is changed. For clarity, we also present separately the maximum $\left\langle S_{a v}\right\rangle^{\max }$ as a function of $\alpha$ in Fig. 7. In the brittle phase $\alpha<\alpha_{c}$, the maximum $\left\langle S_{a v}\right\rangle^{\max }$ is simply the value of the terminal point of the function $\left\langle S_{a v}\right\rangle(\varepsilon)$ at the instant of failure which is practically independent of $\alpha$. However, as $\alpha$ surpasses the critical point $\alpha_{c}$, the coalescence of clusters results in crack sizes which are orders of magnitude larger than those observed in the brittle regime. Inside the ductile regime $\alpha>\alpha_{c}$, the maximum remains constant since it is only determined by the system size $N$ until the weak fibers have a spanning cluster $\alpha_{c} \leqslant \alpha \leqslant \alpha^{*}$. However, for $\alpha>\alpha^{*}$, the maximum of $\left\langle S_{a v}\right\rangle(\varepsilon)$ is reached at saturation, when all the weak clusters have been 


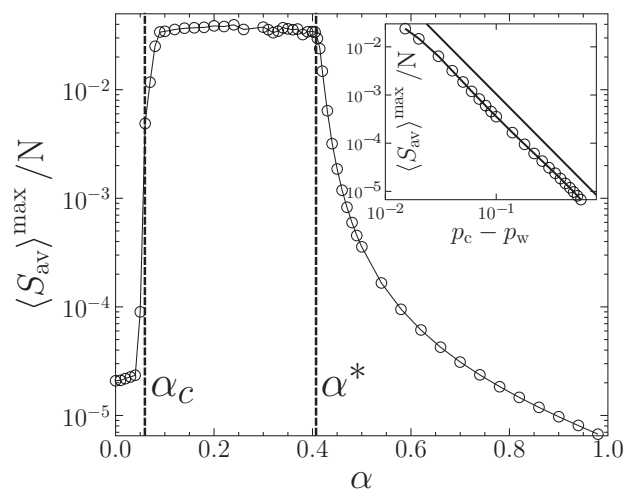

FIG. 7. The maximum value of the average cluster size $\left\langle S_{a v}\right\rangle^{\max }$ averaged over a large number of samples at a given $\alpha$. Three distinct regimes can clearly be distinguished, which are separated by $\alpha_{c}$ and $\alpha^{*}$. The inset presents the same quantity in the range $\alpha>\alpha^{*}$ as a function of $p_{c}-p_{w}$, where $p_{w}=1-\alpha$. The straight line has slope 2.32 .

broken. Hence, in this regime the maximum value $\left\langle S_{a v}\right\rangle^{\max }$ is a decreasing function of $\alpha$ in Fig. 7. It is interesting to note that approaching $\alpha^{*}$ from above $\alpha \rightarrow \alpha_{+}^{*}$ the occupation probability of the weak component $p_{w}=1-\alpha$ tends to $p_{c}$ from below. Consequently, in the regime $\alpha>\alpha^{*}$, the maximum value of the average crack size $\left\langle S_{a v}\right\rangle^{\max }$ has the same critical behavior as the average cluster size of percolation when approaching the critical point

$$
\left\langle S_{a v}\right\rangle^{\max } \sim\left(p_{c}-p_{w}\right)^{-\gamma} .
$$

The inset of Fig. 7 demonstrates that the above prediction is perfectly fulfilled by the simulation data. The numerical value of the exponent $\gamma=2.33 \pm 0.08$ falls very close to the corresponding exponent of percolation $\gamma=43 / 18 \approx 2.39$ [34].

The average number of cracks $\left\langle n_{c}\right\rangle$ also encodes interesting information about the evolution of the crack ensemble. It can be observed in Fig. 6(b) that in the brittle regime $\alpha<\alpha_{c}$, the number of cracks monotonically increases and stops suddenly due to the abrupt failure of the system (compare to Fig. 1). When the fracture is ductile $\alpha \geqslant \alpha_{c}$, the $\left\langle n_{c}\right\rangle$ curves develop a decreasing regime due to the coalescence of cracks such that the position of the maximum $\varepsilon_{m}^{n c}$ marks the configuration where coalescence starts. For clarity, the maximum position $\varepsilon_{m}^{n c}$ is indicated by the vertical lines in Fig. 6 for a few $\alpha$ values. Comparing Figs. 6(a) and 6(b), it can be observed that when the strain $\varepsilon$ exceeds $\varepsilon_{m}^{n c}$ the average cluster size displays a faster growth and reaches its maximum at a larger strain value $\varepsilon_{m}^{S}$. For $\alpha<\alpha_{c}$, these two deformations coincide at $\varepsilon_{m}^{n c}=$ $\varepsilon_{m}^{S}=\varepsilon_{c}^{\mathrm{LLS}}$, where macroscopic failure takes place, however, in the ductile regime the relation $\varepsilon_{m}^{n c}<\varepsilon_{m}^{S}$ holds. Only cracks that develop inside larger clusters of weak fibers can merge, therefore, isolated weak clusters break at higher loads due to the global load reduction associated to the fraction of the load sustained by strong fibers. This morphological feature explains the increase of $\left\langle n_{c}\right\rangle$ at large strains, as displayed in Fig. 6(b). It is important to emphasize that the decreasing branch of $\left\langle n_{c}\right\rangle$ prevails even above $\alpha^{*}$, which shows that merging can occur not only inside the spanning cluster, but even smaller weak clusters may develop several cracks which later on merge.
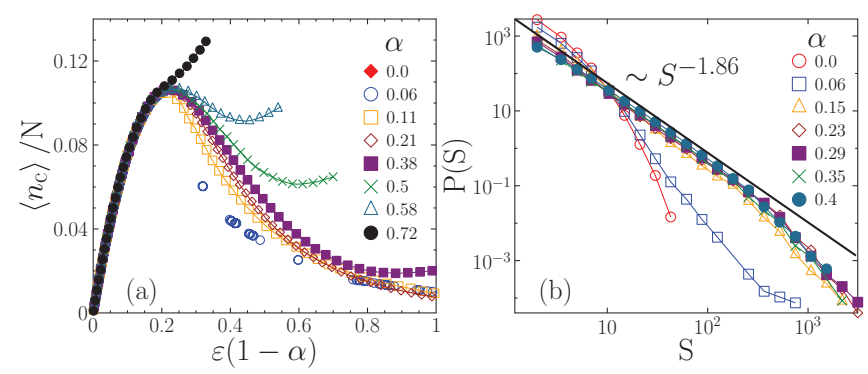

FIG. 8. (Color online) (a) Average number of cracks as a function of $\varepsilon$ rescaled along the horizontal axis with $p_{w}=1-\alpha$. High quality data collapse can be observed up to the maximum. (b) Cluster size distributions determined at $\varepsilon_{m}^{S}$ for several compositions $\alpha$.

The most remarkable result, presented in Fig. 6(b), is that the maximum value of the cluster number remains constant $\left\langle n_{c}\right\rangle / N \approx 0.1$ until the strong component does not have a spanning cluster $\alpha<p_{c}$. In this parameter regime, the maximum cluster number is determined solely by the distribution of the breaking thresholds of the weak fibers. Our result indicates that for a uniform distribution of breaking thresholds, approximately $10 \%$ of the lattice sites serve as nucleation centers of cracks. It is noticeable that until $\alpha<$ $p_{c}$, the presence of strong fibers only shifts the value of the deformation $\varepsilon_{m}^{n c}$ where the maximum is reached. The disappearance of merging is indicated by the monotonicity of $\left\langle n_{c}\right\rangle(\varepsilon)$ which occurs when $\alpha$ exceeds $p_{c}$. In this regime, strong fibers form a spanning cluster and weak clusters are so small that they only nucleate one crack, which gradually grows covering the entire cluster.

In order to quantify the effect of strong fibers on the evolution of the number of cracks, Fig. 8(a) displays $\left\langle n_{c}\right\rangle / N$ as a function of $\varepsilon(1-\alpha)$. A high quality data collapse is obtained up to the maximum, which implies that up to deformations of order

$$
\varepsilon_{m}^{n c} \sim \frac{1}{1-\alpha},
$$

the crack nucleation dominates the behavior of damaged domains, while above $\varepsilon_{m}^{n c}$ crack merging controls the evolution of the microstructure of the system. This behavior implies that large bursts of breaking fibers become relevant for $\varepsilon>\varepsilon_{m}^{n c}$ and they are mainly growth steps of existing cracks. Above the percolation threshold $\left(\alpha>p_{c}\right)$, strong fibers prevent the merging of cracks nucleated within isolated weak clusters. In this case, cracks must nucleate even for $\varepsilon>\varepsilon_{m}^{n c}$ to break all weak fibers, and hence $\left\langle n_{c}\right\rangle$ becomes monotonically increasing.

\section{B. Size distribution of cracks}

The statistics of crack sizes plays an important role in the emergence of localization and macroscopic failure of heterogeneous materials under an increasing external load [17,28,35-37]. In the brittle phase $\alpha<\alpha_{c}$ of our FBM, we evaluated the size distribution $P(S)$ of cracks in the last stable configuration of the bundle before the catastrophic avalanche. It has been discussed above that in the ductile phase $\alpha>\alpha_{c}$, the 

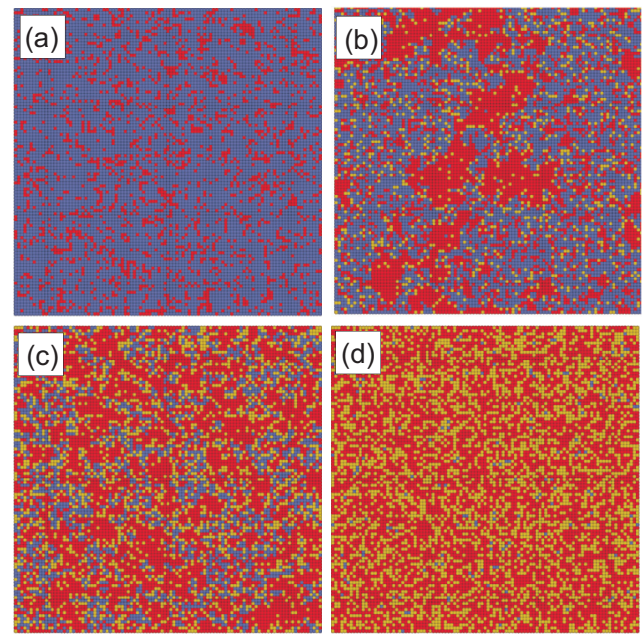

FIG. 9. (Color online) Snapshots of the cluster structure in systems of size $L=100$ taken at the deformation $\varepsilon_{m}^{S}$ where the average cluster size $\left\langle S_{a v}\right\rangle$ has a maximum for different values of the control parameter $\alpha$ : (a) 0 , (b) 0.08 , (c) 0.35 , (d) 0.4 . The assignment of colors is the same as in Fig. 5. When the system only contains weak fibers (a) all the clusters are small compared to the system size. Above the critical point $\alpha_{c}$, growth and merging result in large cluster sizes which are only limited by the system size and by the underlying lattice structure of the weak and strong components.

onset of localization is marked by the position of the maximum $\varepsilon_{m}^{n c}$ of the number of cracks. However, the statistics and structure of cracks at this deformation is rather close to the limiting case of the absence of strong fibers $\alpha=0$. That is why the size distribution of cracks $P(S)$ was evaluated at the deformation $\varepsilon_{m}^{S}$ where the average cluster size $\left\langle S_{a v}\right\rangle(\varepsilon)$ has its maximum.

Figure 9 presents the configuration of the bundle at $\varepsilon_{m}^{S}$ for several values of $\alpha$. It can be observed in Fig. 9(a) that if the bundle only contains weak fibers $\alpha=0$, all clusters are small compared to the system size. Consequently, in Fig. 8(b) the corresponding size distribution $P(S)$ is a rapidly decreasing exponential in agreement with former results on LLS FBMs $[6,17,28]$. In the ductile regime $\alpha>\alpha_{c}$, the spanning cluster covers a large fraction of the system [see Figs. 9(b), 9(c), and $9(\mathrm{~d}))$ ], however, the size of smaller cracks scatters over a broad interval, as well. It is important to note that in these cases the cluster size distribution has a power law regime

$$
P(S) \sim S^{-\tau}
$$

followed by an exponential cutoff. The value of the exponent $\tau=1.86 \pm 0.05$ proved to be lower than the corresponding exponent of percolation $\tau=187 / 91 \approx 2.05$ [34]. It shows that larger cracks more frequently occur due to the correlated growth mechanism and coalescence. It is interesting to note that our results on the size distribution of cracks have a good qualitative agreement with Ref. [35], where a two dimensional fracture model predicted power law distributed crack sizes with exponent $\tau=2$. The authors argued that the growth and coalescence of cracks along the softening regime of the constitutive curve are responsible for the scale free distribution. However, when the failure is brittle or quasibrittle, exponential or log-normal distributions have been obtained in various types of fracture models [17,28,36,38], again in agreement with our results.

\section{DISCUSSION}

We have examined the fracture process of highly heterogeneous materials in the framework of a fiber bundle model. The fibers have identical elastic properties, however, their fracture characteristics are strongly different: a fraction of fibers is strong in the sense that they can sustain any load without breaking, while the rest of the fibers are weak, having statistically distributed strength values. The two components are homogeneously mixed on a square lattice which is then loaded in a stress-controlled way. In order to capture the effect of stress concentration around cracks, we considered localized load sharing such that the load of broken fibers is redistributed equally over its intact nearest neighbors on the lattice. Our computer simulations revealed that varying the fraction of strong fibers $\alpha$ as a control parameter, the mechanical heterogeneity leads to a rich variety of mechanical responses.

Investigating the macroscopic constitutive behavior of the system, we pointed out that at a critical value $\alpha_{c}$ a transition occurs from highly brittle to ductile response. The brittle phase is characterized by the presence of an unstable branch of the constitutive curve along which a macroscopic fraction of fibers breaks in an abrupt avalanche when performing stress-controlled loading. In the ductile regime, the constitutive curve is monotonous with a relatively long plateau regime, which implies that weak fibers break gradually through finite size avalanches leading to stable fracture. The critical fraction $\alpha_{c}$ has an astonishingly small value, showing that adding a very small amount of strong fibers can be sufficient to stabilize an originally brittle system.

On the microscopic scale, our simulations revealed that the interplay of the inhomogeneous stress field arising due to the localized load redistribution and of the load-absorbing effect of strong fibers leads to a rich dynamics. In the ductile regime, the size distribution of avalanches proved to be a power law with an exponent $\mu=2.0$, which is significantly lower than the one of the brittle phase $\mu=\frac{9}{2}$. Approaching the critical fraction from above, the characteristic avalanche size has a power law divergence which shows that the brittle-ductile transition occurs analogous to continuous phase transitions.

The localized load redistribution has the consequence that avalanches of fiber breakings form spatially connected clusters. Since high stress is concentrated along the perimeter of broken clusters, avalanches are typically triggered at these fibers so that avalanches are intermittent steps of the growth of cracks. Our investigations showed that in the brittle regime only small cracks can develop compared to the system size. However, in the ductile phase, the load absorbed by the strong fibers allows the cracks to reach sizes where they merge and form a macroscopic crack spanning the entire system. Merging always occurs until it becomes prevented by the spanning cluster of the strong component in the limit of large values of the control parameter $\alpha$.

Analyzing the statistics of bursts and cracks, we pointed out that at the beginning of the loading process, the nucleation of cracks dominates, while later on coalescence governs the 
evolution of the microstructure. Since the maximum number of cracks does not change, bursts emerging along the plateau of the constitutive curve in the ductile regime are steps of stable crack propagation.

\section{ACKNOWLEDGMENTS}

The work is supported by TAMOP-4.2.2.A-11/1/KONV2012-0036, TAMOP-4.2.2/B-10/1-2010-0024, and OTKA
K84157 projects. The project is implemented through the New Hungary Development Plan, co-financed by the European Union, the European Social Fund, and the European Regional Development Fund. This work was supported by the European Commissions by the Complexity-NET pilot project LOCAT and by NF under the Contract No. ERANET_HU_09-1-20110002. I.P. acknowledges financial support from MICINN (Spain) under Projects No. FIS2008 - $06034-C 02-02$, No. F I S2011-22603, and No. DURSI SGR2009-00634.
[1] M. Alava, P. K. Nukala, and S. Zapperi, Adv. Phys. 55, 349 (2006).

[2] S. Zapperi, A. Vespignani, and H. E. Stanley, Nature (London) 388, 658 (1997).

[3] S. Pradhan, A. Hansen, and B. K. Chakrabarti, Rev. Mod. Phys. 82, 499 (2010).

[4] S. Pradhan, B. K. Chakrabarti, and A. Hansen, Phys. Rev. E 71, 036149 (2005).

[5] R. C. Hidalgo, F. Kun, and H. J. Herrmann, Phys. Rev. E 64, 066122 (2001).

[6] R. C. Hidalgo, Y. Moreno, F. Kun, and H. J. Herrmann, Phys. Rev. E 65, 046148 (2002).

[7] G. G. Batrouni and A. Hansen, Phys. Rev. Lett. 80, 325 (1998).

[8] P. V. V. Nukala, S. Simunovic, and S. Zapperi, J. Stat. Mech: Theor. Exp. (2004) P08001.

[9] G. A. D'Addetta, F. Kun, and E. Ramm, Granular Matter 4, 77 (2002).

[10] F. Raischel, F. Kun, and H. J. Herrmann, Phys. Rev. E 72, 046126 (2005).

[11] P. K. V. V. Nukala, P. Barai, S. Zapperi, M. J. Alava, and S. Simunovic, Phys. Rev. E 82, 026103 (2010).

[12] R. C. Hidalgo, F. Kun, K. Kovács, and I. Pagonabarraga, Phys. Rev. E 80, 051108 (2009).

[13] M. Kloster, A. Hansen, and P. C. Hemmer, Phys. Rev. E 56, 2615 (1997).

[14] F. Kun, H. A. Carmona, J. S. Andrade Jr, and H. J. Herrmann, Phys. Rev. Lett. 100, 094301 (2008).

[15] A. Saichev and D. Sornette, Phys. Rev. E 71, 016608 (2005).

[16] J. V. Andersen, D. Sornette, and K.-t. Leung, Phys. Rev. Lett. 78, 2140 (1997).

[17] S. Zapperi, P. Ray, H. E. Stanley, and A. Vespignani, Phys. Rev. Lett. 78, 1408 (1997).

[18] Y. Moreno, J. B. Gomez, and A. F. Pacheco, Phys. Rev. Lett. 85, 2865 (2000).

[19] K. Kovács, S. Nagy, R. C. Hidalgo, F. Kun, H. J. Herrmann, and I. Pagonabarraga, Phys. Rev. E 77, 036102 (2008).
[20] F. L. Matthews and R. D. Rawlings, Composite Materials: Engineering and Science, 1st ed. (Chapman \& Hall, London, 1994).

[21] R. L. Smith, S. L. Phoenix, M. R. Greenfield, R. B. Henstenburg, and R. E. Pitt, Proc. R. Soc. London, Ser. A 388, 353 (1983).

[22] D. Amitrano, J. Geophys. Res. 108, 2044 (2003).

[23] C. B. Picallo, J. M. López, S. Zapperi, and M. J. Alava, Phys. Rev. Lett. 105, 155502 (2010).

[24] F. Raischel, F. Kun, and H. J. Herrmann, Phys. Rev. E 73, 066101 (2006).

[25] D. Krajcinovic, Damage Mechanics (North Holland, Amsterdam, 1996).

[26] R. C. Hidalgo, K. Kovács, I. Pagonabarraga, and F. Kun, Europhys. Lett. 81, 54005 (2008).

[27] A. Hansen and P. C. Hemmer, Phys. Lett. A 184, 394 (1994).

[28] F. Kun, S. Zapperi, and H. J. Herrmann, Eur. Phys. J. B 17, 269 (2000).

[29] W. I. Newman and S. L. Phoenix, Phys. Rev. E 63, 021507 (2001).

[30] S. L. Phoenix and W. I. Newman, Phys. Rev. E 80, 066115 (2009).

[31] F. Raischel, F. Kun, and H. J. Herrmann, Phys. Rev. E 74, 035104 (2006).

[32] S. Zapperi, P. Ray, H. E. Stanley, and A. Vespignani, Phys. Rev. E 59, 5049 (1999).

[33] F. Bosia, M. J. Buehler, and N. M. Pugno, Phys. Rev. E 82, 056103 (2010).

[34] D. Stauffer and A. Aharony, Introduction to Percolation Theory (Taylor \& Francis, London, 1992).

[35] C. Spyropoulos, C. H. Scholz, and B. E. Shaw, Phys. Rev. E 65, 056105 (2002).

[36] S. Nukala, Phani Kumar V. V. Nukala, S. Šimunović, and F. Guess, Phys. Rev. E 73, 036109 (2006).

[37] K. Z. Nanjo and D. L. Turcotte, Geophys. J. Int. 162, 859 (2005).

[38] A. Stormo, K. S. Gjerden, and A. Hansen, Phys. Rev. E 86, 025101 (2012). 